\title{
HER2/Neu Low Expression
}

National Cancer Institute

\section{Source}

National Cancer Institute. HER2/Neu Low Expression. NCI Thesaurus. Code C156764.

An immunohistochemical result indicating that HER2/Neu (ERBB2) is expressed at low levels in a sample. 